\title{
Experimentation on Analogue Models
}

\author{
S G Sterrett
}

Summary

Analogue models are actual physical setups used to model something else. They are especially useful when what we wish to investigate is difficult to observe or experiment upon due to size or distance in space or time: for example, if the thing we wish to investigate is too large, too far away, takes place on a time scale that is too long, does not yet exist or has ceased to exist. The range and variety of analogue models is too extensive to attempt a survey. In this article, I describe and discuss several different analogue model experiments, the results of those model experiments, and the basis for constructing them and interpreting their results. Examples of analogue models for surface waves in lakes, for earthquakes and volcanoes in geophysics, and for black holes in general relativity, are described, with a focus on examining the bases for claims that these analogues are appropriate analogues of what they are used to investigate. A table showing three different kinds of bases for reasoning using analogue models is provided. Finally, it is shown how the examples in this article counter three common misconceptions about the use of analogue models in physics.

\section{Contents}

Z.1 Introduction

Z.2 Analogue models: terminology and role

Z.2.1 Analogue models and scale models

Z.2.2 Analogue models in philosophy of science

Z.2.3 Analogue models in history of science

Z.3 Analogue models in 21st century physics

Z.3.1 Introduction

Z.3.2 Some recent results

Z.4 Comparing fundamental bases for physical analogue models

Z.4.1 Introduction

Z.4.2 Three Kinds of Bases for physical analogue models

(Table ZT.1 to be inserted as a separate page)

Z.5 Conclusion -- Three Common Misconceptions

Acknowledgments

References

Table ZT.1 -- in a separate file

Figures ZF.1 - ZF.15 -- in a separate file 


\section{Z.1 Introduction}

The array of analogue models used in science is extensive; an attempt to comprehend their range, in size and kind, would have to be abandoned sooner or later. The imagination, intellectual ingenuity, and technical expertise that have been expended in conceiving, constructing and using these various disparate models, each requiring a methodology of construction and deployment appropriate to its nature and use, is dizzying.

Analogue models have been devised and used in physics for quite some time: one of the most common analogies in physics, the analogy between sound and light, was invoked in the midnineteenth century to build a sonic analogue of the Doppler effect for light, which was then used to investigate and establish results for both sound and light. ( [Z.1], [Z.2], [Z.3] ) The analogy was later invoked in the twentieth century to explain Vavilov-Cerenkov radiation, also known as Cerenkov radiation. ( [ Z.4], [Z.3] ) Cerenkov radiation is the electro-magnetic radiation emitted when an electron travels in a medium faster than the speed that light travels in that medium. In his Nobel Lecture, Cerenkov explained: "This radiation has an analogy in acoustics in the form of the so-called shock waves produced by a projectile or an aeroplane travelling at an ultra- sonic velocity (Mach waves). A surface analogy is the generally known bow wave." [Z.4]

More recently, in the twenty-first century, physicists have developed, loosely speaking, analogue space-times and analogue gravity. ( [Z.5], [Z.6], [Z.7] ) Though the initial proposals for analogue models for space-times were based on an analogy between light and sound, once the idea of exploring analogue models of gravity began attracting more interest, a variety of analogue models based on different analogies were proposed. [Z.8] Thus the idea of an analogue based on the analogy between light and sound was expanded to many different kinds of analogues. Faccio points out a commonality that can be seen across all of them, though: all of them can be "reconnected to some form of flowing medium." ( [Z.8]; p. v ) Visser elaborates further: "In all the analogue spacetimes, the key idea is to take some sort of 'excitation' travelling on some sort of 'background', and analyze its propagation in terms of the tools and methods of differential geometry." [Z.9] Arising in part from the interest generated by the work on these analogue models, physicists (Carusotto and Rousseaux) have formulated the notion of a "generalized Cerenkov emission" process. [Z.10]

Another commonly-drawn analogy in physics is the analogy between electrical circuits and mechanical systems. The analogies date from the nineteenth century; it appears they were first invoked to make mechanical models of electrical circuits, the models being seen as a way of 
using knowledge about mechanical systems to provide a better understanding of electrical behavior and concept. ( [Z.11]; [Z.12] ) However, the use of electrical circuits specifically designed to model mechanical systems later became standard: measurements of the flow of current in an appropriately constructed circuit were used to accurately compute quantities used in the mechanical analysis of the corresponding structure; varying elements in the circuit corresponded to varying parameters in the mechanical system, so the effect of differences in a design or a system's initial conditions could be explored. More generally, electronic circuits were used as analogues of anything that could be formalized as a solution of certain classes of differential equations, and ever more sophisticated machines were developed to deal with ever larger classes of differential equations and problems. ( [Z.13]; [Z.14] ; [Z.15], p. 222ff) Other examples of analogues used for computation are mechanical analogues such as the geared devices built in the seventeenth century [Z.16], the soap bubble analogue computers invoking minimization principles that were used to efficiently solve difficult mathematical problems in the twentieth century [Z.17] and biological analogue computers of the twenty-first century such as ameoba-based computing (ABC) analogue models [Z.18].

Other analogue models used experimentally to carry out serious research could be named in astrophysics, cosmology, statistics, economics, geophysics, electromagnetism, fluid mechanics, fluid dynamics, solid mechanics, solid dynamics, structural engineering, coastal engineering, the behavior of volcanoes, and many other fields.

To be clear, these are actual, physical objects or setups, usually human-made, designed to be used as analogue models. The modeling process for employing a physical object or setup as an analogue model includes the identification of a mapping that allows one to correlate something observed or measured in the analogue model with something else (its correlative, such as a corresponding quantity) in the thing modeled. The modeling process also includes a justification of the mapping of some sort, usually invoking a principle or equation to establish the mapping. What is modeled is usually another physical object, process, or phenomenon. The model's limitations in representing certain phenomena in the thing modelled, and any corrections that need to be made due to such limitations, are usually discussed when the analogue model is used for a particular problem. Such qualifications are not meant to undermine or recommend against using the model; they are part of the model and modeling process.

While numerical models implemented on electronic digital computers may have supplanted some of these specific uses, analogue models continue to be used in most of these fields today, and new analogue models and methods of using them continue to be invented and further developed. 
Z.2 Analogue models: terminology and role

\section{Z.2.1 Analogue models and scale models}

It will be helpful to clarify the terminology of analogue model and scale model as used in this article.

\section{Analogue Models}

The word analogue has two connotations relevant in discussions on models: (i) analogous or parallel to; and (ii) continuous, as contrasted with digital. It sometimes happens that a model is analogue according to both meanings. In this article, we will use analogue to mean analogous or parallel to. Thus experimentation on an analogue model is used to mean experimentation on something analogous to the thing modeled. It is important to be clear about what is meant in saying that a model is analogous to the thing modeled.

To say one thing is analogous to another is always to say it is so with respect to a particular analogy, whether or not this is made explicit; there may be many different possible analogies one could draw between two physical things or processes. Thus, just as it does not make sense to ask whether or not one thing is analogous to another without specifying the analogy between them one means to be inquiring about, so it does not make sense to say that one thing is an analogue model of another thing without specifying the analogous relation that is the basis for the correspondences being drawn between the model and what is modeled. Thus, it is implicit in the notion of an analogue model that there is some definite analogous relationship that one means to be referring to, between the model and the thing modelled.

\section{Scale Models}

A scale model (in the sense that engineers and scientific researchers use the term 'scale model') can be considered a special case of an analogue model. (Or, conversely, an analogue model can be considered a generalization of the notion of a scale model.) One way of understanding the relationship between analogue and scale models is by considering how the methodology of physically similar systems applies to each of them.

Using the method of physically similar systems, similarity of two systems is established by showing that each member of a certain (nonunique) set of dimensionless parameters that 
characterizes the behavior of the two systems has the same value in the model as in the thing modelled; in practice exact similarity is often not achievable. [Z.19] Instead, certain of the dimensionless parameters are prioritized, or one aims for the dimensionless parameters to be only approximately equal. That is, it is said that a system $S$ and a system $S^{\prime}$ are similar with respect to a behavior B (e.g., kinematically similar, dynamically similar, similar with respect to buckling behavior, similar with respect to electrical flows, and so on) when a set of dimensionless parameters (ratios) that characterizes that behavior has the same values in S as in S'. Despite the fact that in practice it is often possible to meet this criterion only partially or approximately, the concept of physically similar systems whose similarity is established by dimensional analysis (via establishing equality of the relevant dimensionless parameters), which was originally developed to provide a basis for making use of scale model experiments, still forms the foundation for the use of analogue models and has the virtue that it does not, as most other methods do, require complete knowledge of the equations and conditions that determine the behavior B of interest. [Z.20]

Now, to see the point that a scale model is a special case of an analogue model: each dimensionless parameter is a ratio, so it is only the value of a quantity in relation to other quantities that determines the value of the dimensionless parameters used to establish similarity between two systems $S$ and $S^{\prime}$. To use a simple example, it is Mach number (the ratio of the velocity of a flow or a moving object to the velocity of sound in the medium at the fluid conditions that obtain at a certain time), and not the value of a quantity such as a velocity itself, that indicates whether flow is supersonic or subsonic. The Reynolds number (density $x$ velocity $x$ length, divided by viscosity) is generally indicative of the flow regime (laminar, transitional, or fully developed (turbulent) flow). People often use the term scale model when thinking about scaling linear dimensions in particular, and thus are thinking in terms of ratios of lengths rather than some other (dimensionless) ratio; then, the point about sameness of ratios becomes a point about sameness of ratios of lengths, and hence about the significance of geometrical similarity to the occurrence of some phenomenon. That is, for two systems $\mathrm{S}$ and $\mathrm{S}^{\prime}$, if all we are interested in is a feature or behavior that depends solely on ratios of linear dimensions, then geometrical similarity between model and thing modeled suffices for an object to serve as an analogue model of the thing modeled. This is a special case of a physically similar system in which the relevant dimensionless parameter is a ratio of lengths. [Z.21] A scale model used in architectural layout is a paradigm example of this kind of similarity, and can be considered a special case, even a degenerate case, of a physically similar system. 
Unfortunately, the architectural model as a paradigm of a scale model has become so closely associated with the very idea of a scale model that it can interfere with understanding how broadly the concept of scale model applies. The concept of scale model is not limited to scaling of a linear dimension alone; other quantities can be scaled, too. [Z.21] Despite this, the term scale model is often used in the more restricted sense of scaling the linear dimension of the situation.

Even when restricting the meaning of scale mode/ to the scaling of linear dimensions, it is certainly not necessary that a scale model be smaller in size than what it models, nor even that a scale model have the same geometrical proportions as what it models. Scale models of very small things have been built which are larger than what they model, so as to permit ease in manipulation and observation (e.g., a scale model of a cell); and full size scale models used for prototype testing are common as well (e.g., to test airflow patterns around, or heat convection associated with, a certain shape). Even in full size scale model testing, the method of physical similarity is applicable in designing the experimental conditions to be applied, and in interpreting the results of the experiment. ( [Z.21]; [Z.22] ) The use of distorted scale models calls for some explanation.

\section{Distorted scale models}

Distorted scale models, which are scale models that fail to be geometrically similar to the situation modeled (by design, and in a certain very specific way) [Z.22]; , have been used in scale modeling for over a century; an example may illustrate the nature of, and reason for using, such models.

One example of a distorted scale model is a physical model of Lake Superior [Z.23] that was "built to satisfy the Froude number and Rossby number requirements of dynamic similitude." (The Froude number is indicative of the ratio of inertial forces to the gravity forces of flow, and is important in studies where surface waves are important; the Rossby number is indicative of the ratio of inertial forces to the Coriolis force.) The model was used to generate quantitative results: the coriolis force (in the actual Lake Superior) was modeled by rotating the laboratory model about a vertical axis, and the lake bottom in the model was 'warped' so as to provide the correct scaled depth while the model was rotating. [Z.23; p. 25] The wind flow over the lake was modeled in the laboratory model using a blower with an air distributor. The researchers' experimentation on this analogue model of Lake Superior involved blowing "wind" over it in different directions; they recorded the results in the analogue model by "photographing aluminum particles spread on the water surface." [Z.23] 
The plan view and side view of the model are shown in Figures FZ.1 and FZ.2. The model is a scale model, yet it is not geometrically similar to Lake Superior; the researchers explain:

"Because of the large ratio of horizontal to to vertical distances in Lake Superior, the model was [intentionally made so as to be] vertically distorted." [Z.23] The fact that the vertical linear dimensions of the model are scaled differently than the horizontal linear dimensions are scaled is, of course, taken into account when the corresponding quantities to be associated with the actual Lake Superior are calculated from the values of the quantities observed in the model. The ratio of time in the laboratory model to time in the actual Lake Superior is $1 / 9480$, so that " 1 day in the prototype is equivalent to 9.1 [seconds] in the laboratory model", for instance.

[Image]

Figure FZ.1 Plan View of Lake Superior Distorted Model The ratio of horizontal distance in the laboratory model to horizontal distance in the actual Lake Superior is $1 / 300,000$ [Z.23]

[Image]

Figure FZ.2 Side View of Lake Superior Distorted Model "Because of the large ratio of horizontal to vertical distances in Lake Superior, the model was vertically distorted." The ratio of vertical distance in the laboratory model to vertical distance in the actual Lake Superior is $1 / 1000$. [Z.23]

Such distortion of the vertical dimension in modeling ship performance at sea or fluid behavior in canals is common, but they are not the only use of distorted models. One particularly complex hydraulic model that used distortion was constructed and used to study the impact that large woody debris in a stream had in reconfiguring the creek bed itself. [Z.24] More recently, the use of distorted laboratory models in investigating the structural response of (flexural) plates has been evaluated analytically and judged to provide a reliable means of laboratory investigation. [ Z.25 ]

\section{Z.2.2 The role of analogue models in philosophy of science}

The wide variety of analogue models currently used in serious scientific research mentioned in section Z.1 above is not, however, reflected in the discussions of analogue models one finds in the history and philosophy of science literature. When analogue models are mentioned in philosophy of science, they are usually seen as curiosities suitable for illustrative, entertainment 
or pedagogical purposes, rather than as a serious research methodology. When, on occasion, their role in serious scientific research is recognized, it is usually for a role played in the science of a past era, and often for a qualitative or heuristic purpose at that.

An indication of the extent of confusion and ignorance about analogue models and scale models that exists in mainstream philosophy of science is found in the account in the entry entitled "Models in Science" found in one of the most prominent encyclopedias of philosophy, coauthored by two leading philosophers of science, Roman Frigg and Stephan Hartmann. Scale models are not included under analogical models in that article, and it is claimed that "Typical examples [of scale models] are wooden cars or model bridges." [Z.26] There is no recognition in the article of the notion of physically similar systems or any other methodology of scale models, in spite of the fact that methods of dimensional analysis applied to scale models are the topic of countless books and papers in a wide variety of journals in physics and other scientific disciplines. Instead, the topic of the methodology of scale models is dismissed with the misguided reasoning that "Scale models seem to be a special case of a broader category of representations that Peirce dubbed icons: representations that stand for something else because they closely resemble it" and that "no theory of iconicity for models has been formulated yet." [Z.26] Likewise, the philosopher Ronald Giere, widely recognized in philosophy of science for championing the recognition of the role of models in science, uses examples such as "Watson's original tin and cardboard model of DNA" and "Rutherford's solar system model of the atom" as examples of scale models and analogue models, respectively. ( [Z.27], [Z.28]; p. 747 ) Two well-known discussions in philosophical venues that specifically address experimentation on laboratory water tank analogue models ( [2.29], [2.30] ) do not discuss the foundations of the approach used by researchers who actually employ those models, i.e., dimensional analysis and the method of physically similar systems.

Hence there is little help to be had from the mainstream philosophical literature as far as understanding bases for the scientific reasoning involved in actual experimentation on analogue models by researchers. To be fair, there are a few works that do make some philosophical points about the methodology, the assumptions and the limitations of various bases for experimental methodologies employing analogue models, but they seem unconnected, in that the mainstream discussions in philosophy of science that ought to take note of them seldom do so. ( [Z.31], [Z.32], [Z.33], [Z.34], [Z.35], [Z.36], [Z.19], [Z.37], [Z.38] ). The emphasis in this article will be on the methodologies employed by the researchers who have effectively used laboratory experimentation on analogue models: our interest here is especially in the basis for the inferences drawn using these analogue models. 


\title{
Z.2.3 Analogue models in history of science
}

\author{
Geophysics
}

\section{Analogue models in geophysics: an historical narrative}

An example typical of narratives that view analogue models in terms of their role in the past is historian Naomi Oreskes' "From Scaling to Simulation: Changing Meanings and Ambitions of Models in Geology." [Z.39] According to her narrative, physical scale models were used in the nineteenth and early twentieth century, but in the latter part of the twentieth century, "the word model took on a different meaning: a computer simulation. For earth scientists, this is the dominant meaning it holds today." The main storyline in her narrative has to do with the cause of shifts in epistemic goals of geologists in the late twentieth century. Our interest in this article is simply in the methodology of the analogue models employed. As Oreskes indicates, the method of physically similar systems was applied to the question of how to scale experimental analogue models for the behaviors of interest in studying geologic structure in the 1930 s by M. King Hubbert, in "Theory of scale models as applied to the study of geologic structures." [ Z.40 ] It is a means of obtaining quantitative results about something by taking measurements on an analogue physical model of it.

Hubbert cites Galileo's Two New Sciences, then works by Newton, Stokes, Helmholtz, and Reynolds ([Z.40]; p. 1516 - 1517). Hubbert also describes work done in 1913 (by Koenigsberger and Morath) applying physical similarity to geology. ([Z.40]; p. 1518) Prior to that, many people had built small tabletop models to investigate geological processes, but there was good reason to be skeptical about the validity of these small models; there was a need, Hubbert said, for "an objective criterion to enable one to determine what the correct properties of a model should be for the best similarity, when the properties of the original are known, or whether it is even possible to build a correct model from available materials." Koeningsberger and Morath did "the earliest explicit application of the method of dimensional analysis to tectonic structures"; that was in 1913. ( [Z.40]; p. 1518) So, Hubbert stresses, what he is advocating is not new. Hubbert did not use the more mathematically concise and elegant method of physically similar systems that Buckingham presented in 1914 and which is described elsewhere in this volume [Z.19], but he did use the theory of dimensional analysis, systematically developing and carefully elaborating the dimensionless ratios associated with providing geometrical, kinematical, and geometrical similarity between the model and what is modeled by it. Finding the requirement of dynamic similarity too strict to be practical in many cases, he then goes on to discuss the kinds of 
approximations that are appropriate in special cases. He considers special cases in which inertial forces are very small, ones in which gravitative forces are negligible, and ones in which resistive forces are negligible, explaining which criterion can be violated without affecting the results too much in each case.

As have so many others who have seen themselves as advocates of the method, i.e., as urging the use of the method of physical similarity to handle previously unsolved problems in their profession, Hubbert sounds almost evangelical in his advocacy: ". . the evidence is in that in remote parts of the world the geological professional is already awaking to the importance of so powerful a tool as that afforded by the method of dimensional analysis and correctly made scale models, for the solving of problems that have not yielded satisfactorily to methods of attack previously employed." ( [Z.40]; p. 1519 )

The reasons geophysics needed to use scale models were very much the same as the reasons scale models were being used in other areas such as mechanics and hydrodynamics: the phenomena "are so complicated as a whole as to render complete mathematical analysis difficult or impossible." ( [Z.40]; p. 1460 ) Then "where mathematical analysis is inadequate, and where for one reason or another direct experimentation is precluded, the best remaining alternative is to construct and study a scale model." Writing in 1937, he cites the fields of aerodynamic, hydraulic, mechanical and electrical engineering for their success, then notes that "The geological problems of mountain making and of diastrophism in general are peculiarly of the type that do not lend themselves readily to analysis, and the size of the elements involved place them beyond the range of direct experimentation. In this case also there remains the alternative of studying such phenomena by means of experiments performed upon properly built small scale models." ( [Z.40]; p. 1461 ) In the preface to a later paper "The Strength of the Earth," in which he resolved an apparent paradox in geophysics by appealing to physical similarity, Hubbert explained the value of the approach: "By means of the principles of physical similarity, it is possible to translate geological phenomena whose length and time scales are outside the domain of our direct sensory perception into physically similar systems within that domain." ( [Z.41]; abstract; also quoted in [Z.39]; p. 110] ) He shows the value of developing the characteristics of the materials that would be needed to make a physically similar laboratory model, and shows that even the knowledge of what the physically similar laboratory model would be like is informative. For the purpose of resolving the apparent paradox, understanding scaling relations for the case of the earth is all that is needed: "We learn that the resemblance of the behavior of rocks on a length scale of thousands of miles and a time scale of millions of years is not to that of rocks with which we are familiar but rather to that of the vicscous liquids and weaker plastics of our personal experience." 
( [Z.41]; p. 1653 ) However, the fact that such qualitative lessons can be drawn does not obviate the need for building the models to learn about tectonics in many cases, and even in the same paper in which Hubbert makes the general observation just quoted, he reviews various experimental scale laboratory models that had been built using the method of physical similarity. ( [Z.41]; p. 1653)

Translating geological phenomena occurring 'outside the domain of our direct sensory perception' into that domain is of course extremely significant in the field of geophysics, due to the large sizes and long time scales involved. Given that philosophy has so seldom included this method of experimentation among serious scientific reasoning, the attention Oreskes gives to Hubbert's work on applying physical similarity to geophysics, though not her main point, is a valuable rarity in the literature of history and philosophy of science.

\section{Analogue models in geophysics: the case of volcanology}

One area of geophysics where physical similarity has been employed is volcanology. There are different kinds of volcanoes; volcanoes can differ in configuration and in the mechanisms by which they were formed, for instance. Further, the configurations are seldom static: a given volcano's configuration can change during the process that is of research interest (e.g., eruption, spreading.) Some processes take place over time periods that are very short, involving very high velocities, and others take place over long time periods, e.g., slow changes between eruptions. Concurrent processes are often studied separately in order to understand the mechanisms involved. Sometimes the study focuses on the peculiarities of a specific volcano, and sometimes the subject of the investigation is about general processes and not specific to any volcano. Thus no single example of an analogue model from volcanology is likely to be representative. An example of the use of physical similarity that illustrates how its application can involve very different analogue models of the same volcano is the use of various scaled experiments of different mechanisms involved in the ongoing evolution of Mt Etna in Italy, especially volcano spreading and dike propagation.

To investigate the process of volcanic spreading, cones of sand on layers of sand and silicone were used. [Z.42] Volcanic spreading is a long term process and it involves more than one factor, but the effect of the weight of the volcano on the substratum is one of them. Identifying what the model does not do is part of explaining the model, and the researchers state up front that "Our experiments do not model the effect of the intrusive complexes; they cannot be used as exact scale analogs of volcanoes where the intrusive complexes give the dominant contribution to 
deformation ... " citing other experiments that do so. They add: ". . our experiments do not model the effect of subsidence due to crustal flexure under the load of volcanic edifices" and note that that effect is in fact important for some specific volcanoes and kinds of volcanoes. Their model considers the volcano already cooled, so they are not modeling thermal effects in the experiment, either. Nor, they add, do they take into account "any contribution of magma forces to the destabilization process." [Z.42]

Their explanation of the value of an analogue model of volcano spreading that neglects so many mechanisms is that it can show a relation between the mechanism they wish to model and an effect that can be observed in both the model and the thing modeled: Figures FZ.3 and FZ.4 show the schematic and photographic views of the experiment. Drawing on previous results by others, they use dry sand in the small laboratory model as an analog of brittle rocks in the actual Mt Etna volcano. ( [Z.42]; p. 13,808) The spreading in the model (analogue volcano) experiment takes less than a day; a record of the experiment is made using overhead time lapse photography of the surface of the spreading sand cone. ( [Z.42]; p 13,807)

[Image]

Figure FZ.3 Schematic of experiment of spreading volcano, from initial state of laboratory model (marked 'a') to ten hours from initial state (marked 'c'). From Merle \& Borgia [Z.42].

[Image]

Figure FZ.4 Analogue volcano made of sand, from initial state to end of spreading experiment 10 hours later. From Merle \& Borgia [Z.42], who find the pattern in the end state above 'remarkably similar' to pattern in actual volcano shown in Figure FZ.5.

[Image]

Figure FZ.5 Summit of Mt. Etna volcano (digital elevation model image from Macedonio and Pareschi, University of Pisa) From [Z.42] 
The model is constructed by taking the approach of preserving the dimensionless ratios important to the behavior of spreading, as best they can, and prioritizing some ratios over others. The choice of dimensionless parameters and ratios here is not done from scratch by analysis of the specific problem they are investigating, but draws on Hubbert's analysis of these kinds of problems in geophysics. One interesting aspect of this experiment is that some of the ratios change significantly during the experiment itself. Figure ZF.6 shows the dimensionless variables used. (A note about the notation in the chart in Figure ZF.6: although the Greek letter pi is used to denote dimensionless variables there, not all dimensionless variables indicated in figure ZF.6 have the same role as a dimensionless pi-group or a dimensionless parameter as that term is commonly used in dimensional analyses ( [Z.34], [Z.35], [Z.19] )

[Image]

Figure FZ.6 Average dimensionless numbers for experiments in Merle \& Borgia [Z.42], in actual volcano ('Field') and in laboratory analogue volcano ('Experiment'). Because the configuration changes dramatically during the volcano spreading experiment, some of these ratios change in value during the experiment.

Each experiment is characterized in terms of the values these dimensionless variables take on for that experiment.

The analogue spreading volcano experiment is run using different substratum layers (brittle layer only; brittle layer and ductile layer; ductile layer only) and a buffering solid boundary that buttresses the cone. The experiments are characterized in terms of the values of the dimensionless variables. ( [Z.42]; p. 13,809) The authors remark on the ability of such simple experiments to model features of the natural volcano that had not been modeled before.

More recently, a completely different mechanism suspected to be occurring in the same volcano (although this 'same' volcano, Mt Etna, had erupted in the meantime, including flank (side) eruptions) was modeled by an experiment that modeled a very different kind of mechanism: magma emplacement in the volcano. [Z.43] In this model, a viscous material was injected into a cone of granular material. An aerial view of Mt Etna is shown in Figure ZF.7 The experimental apparatus for the laboratory analogue model used to study the consequences of magma emplacement is shown in Figure ZF.8 
[image]

Figure ZF.7 Mount Etna Volcano, aerial view. The green area is unstable. The fault systems are indicated by RFS, PFS, and TFS. From [Z.43]

[image]

Figure ZF.8 Experimental apparatus used to model magma emplacement by the injection of silicone putty or vegetable oil into an analogue volcano. From [Z.43]

The experimental apparatus and methods used produces measurements of high precision. The size of the model, the materials used in the model, and the scaling of quantities of interest is developed using dimensional analysis, again citing the work of Hubbert. ( Z.43; Section 3.2 )

The research into the mechanisms at work in the Mt Etna volcano using analogue volcanoes is of more than theoretical interest, as thousands of deaths have already resulted from Mt Etna's eruptions. Predicting the future is certainly of interest in the employment of this analogue model; the safety of many could depend upon an understanding of how this specific volcano behaves. The authors note that the use of an analogue model to precisely model the displacements of a specific volcano quantitatively, as was done in their study, is a new use of analogue models in volcanology, and that this work could lead to an 'advanced generation of analog models' that could be compared with those of the actual volcano, and could aid simulation studies. [ Z.43; p. 18-19 ]

Analogue models in geophysics: the lessons of history

Oreskes' narrative, though a welcome rarity in mentioning the historical role of physical similarity, contains a statement about the role of analogue models that could mislead readers into thinking that (or reinforce existing prejudices that) physical analogue models are dispensable in geophysics. As this is a rather common misconception in philosophy of science today, it is useful to confront it here. Oreskes writes "If one could calculate the required properties of materials in a 
scale model, then there was actually no need to build the model itself. One could simply calculate the property of interest." It is not clear what the basis of such a claim could be; building and running experiments with analogue models have been shown to be important in many cases in geophysics from Hubbert's day all the way up to the present. Oreskes continues: "In principle, a computer simulation can be used in precisely the same manner as a mimetic physical model to demonstrate circumstances capable of producing known effects." ( [4.39]; p. 113) This statement gives pride of place to computer simulation in geophysics, which is not deserved. The "in principle" qualification, which is actually an extremely significant qualification, needs to be given sufficient weight.

First, it needs to be emphasized that experimentation on analogue models has not been supplanted by computer simulations; it is surprising how often the misconception that they have been is voiced. Granting that sophisticated computing methods implemented on digital computers are now used for many of the tasks for which analogue models were at one time used, this still does not mean that analogue model experiments are now dispensable. In many cases -perhaps even in most cases --- a great deal more knowledge would be needed in order to construct a computer simulation than would be needed to construct and use an analogue model experimentally in order to yield new knowledge. This is clearly the case in geophysics. Computer simulation is often preferred for reasons of cost and adaptability, but it can not be considered a satisfactory substitute for experimental analogue models in general. Analogue models can be extremely expensive, due to the laboratory personnel and facilities involved in constructing, instrumenting, and carrying out experiments on models with a high degree of precision. Yet, even costly analogue models are still used to this day, as they often reveal phenomena that a computer simulation built using current knowledge does not. This has been as true in other areas such as aeronautics as it has been in geophysics and physical earth sciences: the demise of the wind tunnel has been predicted quite a few times over the last century, but, in spite of such predictions, wind tunnels are still considered indispensable today. So, too, are the analogue models -- some quite costly --- used in geophysics today.

Second, it needs to be emphasized that most computer simulations rely upon information gained by observation and experimentation, especially experimentation on analogue models. The current practice, in geophysics as in so many other fields, is to use both kinds of models in conjunction; over the long term, each methodology can help inform and improve the other. [Z.43] ([Z.44]; p. 1317 ) But there is an asymmetry: while analogue model experiments can be and in the past were performed without benefit of computer simulations, most computer simulations relied heavily on knowledge gained from analogue model experiments --- whether today's users 
of such sophisticated computer packages realize it or not. One practical benefit of computer simulations that accounts for their popularity and widespread use is the ease with which a model can be modified. The advantage of cost and adaptability of computer simulations led to their adoption in cases where the mechanisms were well understood, and this was followed by overreaching claims about what computer simulations were capable of replacing. That these claims were overreaching is seen in retrospect; we can now see that, in geophysics as in many other fields, experimentation on analogue models has not only not been replaced, but still holds an irreplaceable role in investigation.

\section{Role of analogue models vis a vis numerical computer simulation}

These points about the role of analogue models --- i.e., their use in conjunction with, rather than their displacement by, numerical simulation computer methods in the post-computer era --- is readily seen by looking at actual examples of recent research using analogue models. Many examples would serve for this; here we shall look at the details of an example from a recently published (2014) investigation carried out by researchers at Caltech and the University of California and published in a major venue (Journal of Geophysical Research: Solid Earth): the experimental investigation of strong ground motion due to thrust fault earthquakes. [Z.44]

In this investigation, the topic is not the geometry of the changes caused by the earthquakes, but the rupture velocity. Previously, there had been a question of whether the rupture proceeds below or above the velocity at which the seismic shear wave propagates; though observations that supershear ruptures had occurred in natural earthquakes began piling up, their existence went against well established belief. According to Gabuchian et al. [Z.44] , the role of analogue laboratory models in the discovery and acceptance of the occurrence of a high speed "rupture velocity" that does exceed this critical velocity was profound: they write that "it was the experimental discovery of supershear ruptures occurring repeatedly and reproducibly under highly instrumented and controlled laboratory conditions ... that stimulated the recent flurry of theoretical activities on the subject." They report that the theoretical activities were themselves changed in important ways as a result of the laboratory results; "it motivated seismologists to remove [certain speed restrictions], to revisit a number of historic earthquake events, and to reexamine irregular field observations in search for such a phenomenon." [Z.44]

Gabuchian et al. argue that analogue experiments are crucial in developing and validating numerical models [Z.44], and that, even more importantly, they are: 
"the only way to provide fresh observations of previously unknown phenomenon (discovery) that can then be investigated in numerical models and in seismological data. Indeed, many of the effects of dip-slip faults [. . . ] were predicted by the analog foam rubber models of Brune [1996]. Thus, laboratory experiments, numerical models, and seismic observations can be used together and iteratively to more fully investigate the physics of faulting." ( [Z.44]; p. 1317 )

Gabuchian et al.'s 2014 earthquake investigation [Z.44] uses an analog material called homalite, which is a high density photoelastic polymer material. The material is prepared and the setup instrumented in such a way that it provides a high-precision model of laboratory earthquakes, with respect to the quantities measured. The results are also visually accessible. Many analogue models in geophysics use materials more like the material modeled, as in the volcanic spreading example. The use of a photoelastic polymer such as homalite in which shocks are caused thermally or electrically --- rather than being mechanically-induced --- reflects both the recent development of new laboratory techniques and the recognition that fundamentally different processes may need to be studied now in order to understand the consequences of the phenomena of dynamic fracture. Experimental reproducibility is addressed in their studies, too. ( section 4.3 of [Z.44] ) The interest here is definitely not limited to explaining the past, but in using analogue models to understand the future as well as the past.

A sketch of the experimental specimen used in Gabuchian et al.'s 2014 experiments is shown in Figure ZF.9.

[image]

Figure ZF.9 Experimental Specimen made of homalite (a high density photoelastic polymer material). A dynamic rupture is triggered at the interface; the upper surface on the right side of the interface serves as a 'simulated Earth's surface.' From [Z.44]

As indicated in Figure ZF.9, one of the surfaces of the specimen serves as a simulated 'earth's surface.' The design and sizing of the specimen involved some mathematical analysis; avoiding reflected waves and buckling were two requirements that had to be met. The photoelastic images revealing the difference between the experimental results in the subcritical and supercritical cases are very striking and shown in Figure ZF.10. We shall not go into the details of the results here, except to point out the Mach cone in the supercritical case (on the right hand side of Fig. ZF.10), and that one can visually compare the photoelastic image of the supercritical case to that of the photoelastic image of the subcritical case (left side of Fig. ZF.10). 
[image]

Figure ZF.10 Photoelastic images of experimental results. A dynamic rupture is induced on the diagonal surface so that the propagation of the rupture in the homalite specimen can be studied. The Mach cone is clearly visible in the right hand image. From [Z.44]

The significance of this research for our purposes is that, as described above, this research using analogue volcanoes shows a significant feature, or phenomenon, that was not uncoverable using existing methods of analyzing earthquakes. [Z.44]

\section{Z.3 Analogue models in physics}

\section{Z.3.1 Introduction}

Analogue models are "a constant presence in the world of physics and an invaluable instrument in the progress of our knowledge of the world that surrounds us," in the words of the editors of a recent (2013) collection on the use of analogue models in contemporary theoretical physics [Z.8], though, as they point out, it "would be impossible to give a comprehensive list of these analogue models." ( [Z.8]; p. v ) One especially interesting example in theoretical physics is the use of analogue models of spacetime briefly mentioned in the opening of this chapter. A variety of such analogue models have been proposed, including analogues that employ surface waves, BoseEinstein condensates, graphene sheets, optical fibres, optical glass, and laser pulse analogues. Some of these have so far only been used in probing questions about gravity and spacetime theoretically, but some have also been used to actually construct analogue models in the laboratory. [Z.8]

Lessons from the Nineteenth Century

The use of analogue models in investigating cosmology, e.g., analogue spacetimes, or analogue gravity, may seem quite distant from the more familiar analogue models in geophysics and nineteenth century mechanics, but, conceptually, it actually looks like a most natural outgrowth of them. In the late nineteenth century, while engineers were developing similarity methods to improve their designs of and predictions about ships and structures, physicists explicitly employed analogies to help them think through theory and come up with experiments about light, heat, sound, electricity, and magnetism. One of the most well-known of these was the analogy 
between light and sound that was based on the fact that both were waves; another was the analogy between fluid, heat and electrical currents that was based on the fact that the partial differential equations describing all three such 'flows' were of the same form.

\section{Sound as an analogue of light: the power of experimentation on analogues}

The analogy between light and sound was especially fruitful in the development of the correct understanding of the Doppler effect. (The Doppler effect is the change in frequency observed due to relative motion between the source of a wave and an observer. If the relative motion between source and observer is towards each other, the observed frequency increases; if the relative motion between source and observer is away from each other, then the observed frequency decreases.) To put it more precisely, the relevant factor is the ratio of the velocity of the relative motion between observer and source to the velocity $\mathrm{c}$, where $\mathrm{c}$ is the velocity of sound for a change in pitch, or the velocity of light for colour shift. Because the velocity of light is so high, the velocity of motion required to create an observable change in pitch is much, much less than that required to create an observable change in colour. ( [Z.3]; p. 18 ) Mach devised and carried out laboratory experiments in which changing the relative motion between the observer and the sound source resulted in changes in observed frequency [Z.2], concluding in 1860 that 'the fluctuation in the pitch is dependent on no other circumstance than the direction and speed [of the source] with respect to the observer.' [Z.1], ( [Z.3]; p. 18)

Could the laboratory experiments using sound be considered an analogue for light in a serious scientific sense? Eventually, Mach became convinced that they could be; in 1878 he published an article on it, no longer hesitant about extending the Doppler principle from his experiments on sound to the realm of optics. The Doppler effect for light, he argues, follows from the characteristics of light that are common to both sound and light waves. He does not need to assume that light is a mechanical wave in order to extend his results from sound to light. The characteristics that light and sound have in common that are relevant to applying the Doppler principle are things such as: being propagated in time with a finite velocity, having spatial and temporal periodicity, and being able to be algebraically summed. This characterization does not assume the existence of a medium for light. Mach considers the experiments on sound to be confirmatory for light, and argues that on the basis of them he can conclude with confidence that the Doppler principle applies to light. [Z.1], [Z.3]

To lay out the reasoning that Mach eventually uses in claiming that his experiments on the Doppler effect for sound waves in the laboratory are confirmatory of the Doppler effect for light 
propagation in astronomy: His theory is that it is the relative motion of wave (or signal) source and observer that is responsible for the Doppler effect. Mach identifies the characteristics common not only to light and sound, but to all oscillatory motion, that he believes are sufficient for the occurrence of the Doppler effect. He shows that any oscillatory motion having these characteristics will give rise to the Doppler effect, according to his line of reasoning. The mechanism does not matter; he is explicit about this point, in part because he wishes to emphasize that the existence of the Doppler effect for sound does not depend on features of the medium of transmission. Mach then experimentally confirms that, as his reasoning predicts, the Doppler effect arises for sound in a laboratory setup that allows him to manipulate the relative motion of signal source and observer. Based upon the fact that light has in common with sound those characteristics he has shown are responsible for giving rise to the Doppler effect according to his line of reasoning, he concludes that the experimental confirmation of his experiments for sound in the laboratory apply to light in astronomical observations. [Z.3] As we shall see, this kind of approach using analogue models is very like approaches still in use in physics today.

Water as an analogue of electricity: limitations of generalizing from analogues

The nature and limits of analogical reasoning, including reasoning about experiments on analogues, was also a concern of nineteenth century physics. In a paper entitled "On Discontinuous Movements Of Fluids" (which is discussed in more detail in [Z.19], in this volume), Helmholtz points out both the invaluable role that analogue models can fulfill, and the limitations they may display.

As for the limitation of analogical reasoning on the basis that two things instantiate the same equation, Helmholtz notes that "the partial differential equations for the interior of an incompressible fluid that is not subject to friction and whose particles have no motion of rotation" are precisely the same as the partial differential equations for "stationary currents of electricity or heat in conductors of uniform conductivity." [Z.45] Yet, he notes, even for the same configurations and boundary conditions, the behavior of these different kinds of currents can differ. ([Z.45]; p. 58) The explanation he gives is that in some situations, "the liquid is torn asunder", whereas electricity and heat flows are not. Based upon observations, the difference in behavior between fluid currents on the one hand and electrical and heat currents on the other is due to "a surface of separation" that exists or arises in the case of the fluid. 
Helmholtz identifies another method:

In this state of affairs [the insolubility of the hydrodynamic equations for many cases of interest] I desire to call attention to an application of the hydro-dynamic equations that allows one to transfer the results of observations made upon any fluid and with an apparatus of given dimensions and velocity over to a geometrically similar mass of another fluid and to apparatus of other magnitudes and to other velocities of motion." ( [Z.46]; p. 68)

The method Helmholtz is referring to, which he presented in this now-classic paper (originally published in German in 1873), thus differs from deducing predictions from theory. The method he presents there does make use of the fact that the same equation applies to both situations to provide a basis for using one situation as an analogue for another. However, Helmholtz derives the dimensionless parameters that must be made the same between analogue model and what is modelled. The topic is discussed in more detail in [Z.19]. What Helmholtz describes is a special case of a scale model, for he specifies that the bodies are to be geometrically similar, and involve fluids to which the hydro-dynamic equations apply. This is a use of an analogue model in which the basis for drawing the analogy, although it makes use of the fact that there is an equation instantiated by analogue and thing modelled, does not rely on that fact alone: there are dimensionless parameters that must be held the same between analogue and thing modelled.

This kind of concern about basing the use of an analogue model on the fact that the analogue model and what it models both instantiate the same equation is reflected in critiques of their use in physics today. The concern Helmholtz raised in the nineteenth century (about the surfaces of separation that arise in fluid flow) regarding the limitations of analogues between different kinds of flow (heat, electrical, hydrodynamic) arises today not only when there is a difference in material or what is flowing, but also when the scales between laboratory model and what is modeled are so different that one cannot assume that the same forces or mechanisms are at work in analogue model and what is modelled.

Whether the physicists using analogue approaches in twenty-first century physics realize it or not, the new methods they are developing and the concerns they are raising about them have precursors in the nineteenth century. 


\section{Z.3.2 Some recent results using analogue models}

The use of analogue models of space-time in the twenty-first century involves drawing analogies between flows of various sorts, too. However, the interest does not seem to be historically continuous with the nineteenth century efforts.

Unruh's 1981 "Experimental Black Hole Evaporation?"

The current interest in analogue models of gravity is usually traced to a paper by William Unruh published in 1981. In that very short paper, Unruh addressed what he called "one of the most surprising discoveries of the past ten years": black hole evaporation. He noted that "experimental investigation of the phenomenon would seem to be virtually impossible, and would depend on the highly unlikely discovery of a small black hole (a relic of the initial stages in the life of the universe perhaps) of the earth." However, he said, "a physical system exists which has all the properties of a black hole as far as thermal radiation is concerned, but in which the physics is completely understood." [Z.47]

The physical system he referred to was a sound wave propagated in supersonic flow. He restricted consideration to cases of "the background fluid smoothly exceeding the velocity of sound" which, he notes, can be assured by the use of "a suitably shaped nozzle." ( [Z.47];, p. 1352 and n. 7 ) Indeed, such a "suitably shaped" nozzle exists; the de Laval nozzle was invented in the nineteenth century for steam applications, and is used in rocket design and many other applications today.

[image]

Figure ZF. 11 DeLaval Nozzle from [Z.48]; p. 380.

This insightful invention can be used to create conditions for smooth supersonic flow (i.e., a region in which flow is supersonic but a shock wave does not occur). ( [Z.48]; p. 380)

Normally, in a pipe or convergent (decreasing cross sectional area) nozzle, once flow reaches the critical flow, or "choked" conditions, the velocity of the flow at the throat of the nozzle does not increase, even if the pressure upstream increases. However, if the convergent nozzle has an appropriately designed divergent nozzle attached to its outlet, it is possible for the velocity of the 
flow to increase in the divergent (increasing area) portion of the nozzle, after passing through the throat (minimum area) of the nozzle. The striking thing about this situation (as many engineers are aware), is that changes in the downstream pressure do not affect the rate of flow in the nozzle. Considering the phenomenon in terms of pressure signals, one way to think of this is that the information that the pressure downstream has changed (i.e., a pressure signal or pressure pulse) cannot travel back upstream to the throat of the nozzle. [Z.49] This feature of flow in a DeLaval nozzle is depicted in Figure ZF.12

[ image ]

Figure ZF.12 Scheme of a DeLaval Nozzle showing important features of flow behavior. From ( [Z.49]; p. 89 )

This physical situation is an analogue model, in that, as Unruh put it: "The model of the behavior of a quantum field in a classical gravitational field is the motion of sound waves in convergent fluid flow" which, he added "forms an excellent theoretical laboratory where many of the unknown effects that quantum gravity could exert on black hole evaporation can be modelled . . . the phonons emitted are quantum fluctuations of the fluid flow and thus affect their own propagation in exactly the same way that graviton emission affects the space-time on which the various relativistic fields propagate." ([Z.47]; p. 1353) He had some doubts about how detectable the emission would be in that physical system, though.

Reasoning about analogues of gravity before and after 1981

Visser notes that, actually, notions of analogues of gravity "have, to some extent, been quietly in circulation almost since the inception of general relativity itself" citing Walther Gordon's introduction of "a notion of 'effective metric' to describe the effect of a refractive index on the propagation of light" and "notions developed in optics to represent gravitational fields in terms of an 'equivalent refractive index.' " [Z.6] Max Born's sonic analogue of the kinematics of special relativity might be cited as another example of sorts:

'[...] if we use sound signals to regulate the clocks, Einstein's kinematics can be applied in its entirety to ships that move through motionless air. The symbol c would then denote the velocity of sound in all formulae. Every moving ship would have its own units of length and time according to its velocity, and the Lorentz transformations would hold between the 
systems of measurement of the various ships. We should have before us a consistent Einsteinian world on a small scale' ( [Z.50]; p. 251, also quoted and discussed in [Z.3] ).

Nonetheless, Unruh's short 1981 paper is regarded as marking a new era in the revival of analogue notions of gravity, although the thinking concerning the analogue involved in it has progressed since then. Whereas, in the 1981 paper, Unruh had proposed an analog involving sound traveling in a fluid flowing through a nozzle, by 2002, Schutzhold and Unruh [Z.51] proposed "gravity waves of a flowing fluid in a shallow basin" as an analogue to study black holes in a curved space-time. The surface wave setup permitted manipulations not available in the earlier proposal, due to being able to alter the depth of the water. The reasoning Schutzhold and Unruh use here appeals to "similar equations", i.e., that the behavior of interest in the analogue and the behavior of interest in what the analogue models are described by equations of the same form.

"Analogs, which obey similar equations of motion to fields around a black hole raise the possibility of demonstrating some of the most unusual properties of black holes in the laboratory. This is the basic idea of the black and white hole analogs [ . . ] originally proposed by Unruh [in 1981] . . . The sonic analogs established there are based on the observation that sound waves in flowing fluids are (under appropriate conditions) governed by the same wave equation as a scalar field in a curved space- time. The acoustic horizon, which occurs if the velocity of the fluid exceeds the speed of sound within the liquid, acts on sound waves exactly as a black hole horizon does on, for example, scalar waves. [emphasis added]" [Z.51]

However, there is also reasoning about aspects of the analogue model (surface waves in fluid) that is not part of the analogy, in a way that aims to show how the fuller knowledge we have about the analogue model might be drawn upon:

"In the case of a fluid, one knows that the fluid equation of motion is inapplicable at high frequencies and short wavelengths. At wavelengths shorter than the interatomic spacing, sound waves do not exist and thus the naive derivation of the temperature of [sonic analogues of black] holes will fail. But unlike for black holes, for [sonic analogues of black] holes, the theory of physics at short wavelengths, the atomic theory of matter, is well established. For black holes, a quantum theory of gravity is still a dream. Thus, if one could show that for [sonic analogues of black] holes the existence of the changes in the theory at short wavelengths did not destroy the existence of thermal radiation from a [sonic analogue of a black] hole, one would have far more faith that whatever changes in the theory quantum gravity created, 
whatever nonlinearities quantum gravity introduced into the theory, the prediction of the thermal radiation from black holes was robust. " ( [Z.52]; p. 2908)

This is basically an attempt to identify the relevant characteristics of a system of which the thermal radiation is a consequence. The approach is reminiscent of Mach's approach in investigating the Doppler effect: if features other than the relative motion of source and observer did not make a difference to the existence of the Doppler effect, that would increase confidence (or show to those who were sceptical) that the Doppler effect depended only on the relative motion of source and observer. In this case (sonic analogues of black holes), if features other than those related to instantiating the equation that the black hole and the sonic analogue for it had in common seemed not to make a difference to the existence of a certain effect, then one's confidence that the effect followed in virtue of a system instantiating the equation would be strengthened. But it could go the other way, too:

On the other hand, if the introduction of the atomicity of matter invariably destroyed the thermal radiation for [sonic analogues of black] holes, one would strongly suspect that the thermal nature of black holes would not survive the complications introduced by quantum gravity. ( [Z.52]; p. 2908 )

Unruh had shown concern from the start that Hawking's derivation of black hole evaporation relied upon assumptions he describes as 'absurd.' There was thus value in being able to sort out which features or characteristics of the situation are actually responsible for the Hawking effect. Visser had argued in 1998 [Z.53] that Hawking radiation is a kinematic effect independent of dynamics; In Schutzhold and Unruh's 2002 paper, they remark on what this means for the value of analogue models of gravity:

" Although the kinematics of the waves propagating within the black and white hole analogs are governed by the same equation as those in a curved space-time, the dynamics of the effective metric itself are not described by the same laws as gravity (i.e. the Einstein equations) in general.

In this way the analogs allow one to separate the dynamical effects of gravity (following from the Einstein equations) from more general (kinematic) phenomena." [Z.51]

Our interest here is in the methodologies that are used to underwrite the use of analogue models to serve as models of what they are used to model. However, it is worth noting that this use of analogue models -- i.e., using them to help sort out what the phenomenon of interest is 
dependent upon for its existence -- is a valuable help that analogue models can provide when there is dispute about the dependency. It is not a new kind of reasoning, of course, for we saw that Mach used it in his experiments on the Doppler effect, especially to argue against a view held by others (e.g., Petzval) that the phenomenon arose from features of the medium of transmission. Nor does reasoning about sorting out dependencies necessarily require the use of analogue models or experimentation; mathematicians often show that a result can be proven with fewer assumptions than currently-known proofs, without resorting to laboratory experiments to do so.

Sorting out dependencies: "Measurement of Stimulated Hawking Emission in an Analogue System"

When Hawking radiation was finally measured in an analogue model of a black hole, it was for exactly this benefit -- sorting out dependencies -- that it was especially valued, and the interest was in observing the existence of the phenomenon. Unruh argued for his view that the result of the experiment counted as a genuine measurement of Hawking radiation in 2010; there are also comprehensive reports on the experiment by the experimental team headed by Silke Weinfurtner. [Z.54] [Z.55] The laboratory setup is shown in Figure ZF.13 ( Figure 8.2 of [Z.55] ): a region of high-velocity flow, including (surface wave) horizons, is created by placing a streamlined obstacle in the water flume. ( Figure 8.4 of [Z.55] )

[image]

Figure ZF.13 Experimental Apparatus in Weinfurtner et al. analogue model of Hawking emission. From [Z.55]: "The experimental apparatus used in our experiments: (1) holding reservoir, (2) pump and pump valve, (3) intake reservoir, (4) flume, (5) obstacle, (6) wave generator, and (7) adjustable weir."

[image]

Figure ZF.14 Obstacle in Weinfurtner et al.'s experiments. From [Z.55]: "(1a) and (1b) curved parts motivated by airplane wing; (2) flat aluminum plate to further reduce flow separation; and (3) flat top aluminum plate to reduce wave tunneling effects."

Against this flow, long waves are propagated, which become blocked and converted into short waves, thus creating a laboratory analogue of the behavior of interest: 
It is this blocking of the ingoing waves that creates the analogy with the white hole horizon in general relativity. That is, there is a region that the shallow water waves cannot access, just as light cannot enter a white hole horizon. Note that while our experiment is on white hole horizon analogues, they are equivalent to the time inverse of black hole analogues. ( [Z.54]; p. 120312-2)

Rousseaux illustrates the effect occurring in nature: Figure ZF.15 below, of a white hole formed where a river enters the sea, is one such example from his work [Z.56]. Other examples are fluid being poured into a sink, and a whale's "fluke-print": "As a whale swims or dives, it releases a vortex ring behind its fluke at each oscillation. The flow induced on the free surface is directed radially and forms an oval patch that gravity waves cannot enter ... " [Z.56; p. 99]

[image]

Figure ZF.15 White Holes in Nature. Where a river enters the sea, the sea waves may be 'blocked.' From [Z.56]

In the 2011 paper by Weinfurtner et al., "Measurement of stimulated Hawking emission in an analogue system", the authors note that they already had numerical studies indicating that "the [Hawking] effect is independent of short-wavelength physics." The motivation for experimentation on the analogue model is that, were they to be able to show that there was thermal emission in their physical setup, this would "indicate the generic nature of the Hawking thermal process." This is because the water tank/flume physical system "exhibits turbulence, viscosity, and nonlinearities"; their argument seems to be that the existence of thermal emission in spite of these would show that the process is a feature that follows from the wave kinematics of the physical setup, not forces arising due to these other features of the setup. And, if that were true, the result would be a very general one, applying to waves of any sort.

The measurement result is not as simple as providing the value of a quantity; the researchers identified a certain dimensionless parameter having to do with the ratio of the amplitudes of the waves that were absorbed to those that were emitted, which is important in describing thermal (Hawking) emission, and they investigated how it scaled with frequency. This was a check that what they were observing did have the character of the theorized emission, i.e., this ratio scaled with frequency in just the way one would expect thermal (Hawking) emission to. 
The role of the results from the analogue model do not have their significance as stand alone results; as Weinfurtner et al. point out, there are certainly other, theoretical reasons for suspecting the thermal emission to be independent of quantum gravity or Planck-scale physics. There are other reasons for departing from the view Hawking had when he first discovered it, i.e., for abandoning the view that thermal emission was "a feature peculiar to black holes" ( [Z.55]; p. 179), notably Visser's work in 1998 mentioned above, in which he argued that Hawking radiation is a "purely kinematic effect" that is "generic to Lorentzian geometries containing event horizons." [Z.53], [Z.57] The experimentation on an analogue model demonstrated how to create and measure such thermal emission in a laboratory setup with classical waves, lending support to the theoretical conclusions that the phenomenon is a far more general feature of waves in systems of a certain sort. Weinfurtner et al close the paper in which they report their results with an indication of even more valuable work that experimentation on analogue models of black holes might do:

"It would still be exciting to measure the spontaneous emission from a black hole. While finding small black holes to test the prediction directly is beyond experimental reach, such measurements might be achievable in other analogue models, like Bose Einstein condensates, or optical fibre systems. [citing such models being developed by others.]" [Z.54 ]

\section{Z.4 Comparing fundamental bases for physical analogue models}

\section{Z.4.1 Introduction}

Analogue models are often described in terms of an equation that both a physical model and what it models have in common; on such an account, an analogue model is described as one of two physical setups instantiating a certain equation. Common examples are a hydraulic system and an electrical system (each of which can serve as an analogue model of the other), or an oscillating electric circuit modeling an oscillating mechanical device. This is certainly one kind of basis for an analogue model. However, as the discussions in this article drawing on actual scientific practice indicate, instantiating the same equation is not always the bases used to develop, justify, and reason using analogue models. The actual logic involved is often far more sophisticated, and sometimes does not require as much knowledge about the phenomenon one wished to bring about as the approach of exhibiting an equation that is instantiated by both the model and the thing modelled does. Gathering together the insights above, along with points made in the sources cited, three kinds of bases for analogue models can be identified. These are depicted in the table below (Table ZT.1) Besides the account just mentioned, which is listed 
below as 'Equation in Common,' there are two others: 'Characteristics essential to the behavior of interest are the same in the model as in the thing or system modeled'; and 'Physically Similar Systems.'

Z.4.2 Three Kinds of Bases for physical analogue models

These three kinds of bases are listed below and the points made about them are organized in Table ZT.1.

--- INSERT TABLE ZT.1 APPROXIMATELY at this point in the paper ----

(Table ZT.1 uses landscape view orientation so it requires an entire page of its own)

Equation in common

When the basis for the analogy between the analogue model and what is modeled is an appeal to the fact that an equation governing a quantity or phenomenon in the model is the same equation governing the corresponding quantity or phenomenon in the thing modelled, we will say the basis is having an equation in common. The equation may refer to different physical things, processes, or systems in the analogue model than in what is modeled.

To illustrate with an example using a hydraulic circuit as an analogue of an electrical circuit, experimentation on the analogue model (e.g., hydraulic setup) is used to inform the researcher about what will happen in the analogous setup modeled (e.g., the electrical circuit). The knowledge relied upon in constructing and using the model is the partial differential equation of fluid flow governing the hydraulic flow behavior in the analogue model, the analogous equation for electrical current in the electrical circuit, and that they can be put in the same form so as to permit drawing correspondences between the fluid quantities (e.g., flow velocity, pressure) and electrical quantities (e.g., current, voltage).

This kind of basis has a potential vulnerability: the behavior of analogue model and thing modeled can diverge. A key example of this is due to Helmholtz: "On Discontinuous Movements Of Fluids" (1868) in which a 'surface of separation' arises in the case of fluids, but not in the case of electrical flows -- even though the partial differential equation takes the same form for both flows. [Z.45] Nevertheless, there are cases for which the discontinuity Helmholtz cites is known 
not to arise (i.e., the pressure does not become negative in the case of interest). Which cases are which is one piece of knowledge relied upon (or, sometimes, explored) when using this kind of basis for the analogue model.

\section{Relevant Characteristics in Common}

When the basis for the analogy between the analogue model and what is modeled is the fact that the characteristics essential to the behavior of interest are the same in the model as in the thing or system modeled, we will call it a case of Relevant Characteristics in Common.

Sometimes the relevant characteristics are derived from equations and/or analyses of mechanisms, so the knowledge relied upon may involve some of the same information that is relied upon in the 'equation in common' approach; it depends upon the reasoning the researcher uses to decide which characteristics of the situation are relevant. Sometimes a researcher may employ an approach based on partial knowledge (i.e., less knowledge than that needed to solve the problem) -- but the partial knowledge may be enough to identify what the characteristics relevant to producing the behavior of interest are. Or, the partial knowledge may be enough to show that an analogue model and what it models will display the same behavior of interest, so that experimenting on the analogue is informative about how what it models will behave. Some of the scaling arguments used in developing the analogies underwriting models of analogue gravity are examples of this, as in Rousseaux's discussions of the differences between shallow and deep water tank analogue models. [Z.56] Rousseaux's scaling arguments are based upon dimensional analysis, though he does not lay out and solve the problem as one of physically similar systems.

Other examples of this sort are Ernst Mach's work on the Doppler effect and Weinfurtner et al.'s work on identifying the classical features of the Hawking process: E. Mach identified characteristics essential to being a wave that did not depend upon the existence of a medium of transmission (spatial \& temporal periodicity, finite velocity, can be algebraically summed) \& showed the Doppler effect due to relative motion of source \& observer was a consequence of these features, then verified by experiments on analogue model. [Z.1] [Z.3] Weinfurtner et al. identified classical features of the Hawking process that did not depend upon quantum gravity or Planck-scale physics (e.g., wave pair formation), then showed Hawking radiation a consequence of these features of waves, and subsequently verified by experiment that the analogue of Hawking radiation occurred on the analogue model.) [ Z.54], [Z.55] 
Physically Similar Systems

When employing the method known as physically similar systems, a (nonunique) set of dimensionless parameters that characterizes the system with respect to a certain kind of behavior is identified using the method of dimensional analysis; similarity of system behavior between $\mathrm{S}$ and $S^{\prime}$ is established when these parameters have the same value in $S$ as in $S^{\prime}$ [ see [Z.19], this volume] The knowledge relied upon for this method is the knowledge as to which quantities are relevant to the behavior of interest. Generally this is less information than is required in the 'equation in common' method. In a much more fundamental sense of reliance, the researcher is also relying on a basic assumption made implicitly in much of scientific research: that the behavior is rule-governed, in that it is assumed that there is a relation (possibly unknown) between the quantities relevant to the behavior of interest, and that the relation can be expressed by a physical equation.

\section{Z.5 Conclusion -- Three Common Misconceptions}

The discussion presented here, of a few selected examples of analogue models and the investigation of the different bases for their use, should help put to rest three common misconceptions about the use of analogue models in physics today.

First, the misconception that analogue models are a thing of the past: As the examples discussed above indicate, analogue models are not a thing of the past. In fact, there are new areas of application and new kinds of analogue models being developed; the recent surge in development of analogue models in general relativity is one striking example. [Z.57] [Z.5] Yet, the use of some of these models in the 21 st century does have precursors in the nineteenth century.

\section{Second, the misconception that analogue models serve merely illustrative or pedagogical} purposes. Many of the examples described above are cases of serious investigative research.

This is so even when the main benefit of the model is gaining a better qualitative understanding of the mechanisms at work. Rousseaux remarks that the investigation of analogue gravity "through the prism of water waves theory has broadened our definition of a horizon" ( [Z.56]; p. 106 ) In geophysics, experimentation on analogue models has sometimes brought about an appreciation of mechanisms that might be at work and ought to be investigated, which is a kind of discovery. [ Z.44 ] 
Third, the misconception that numerical methods along with high-speed digital computers can always provide whatever an analogue model could provide. This is the most pernicious and deep-seated of the three misconceptions. It betrays a fundamental misunderstanding of the logic behind analogue models. Such statements are probably based on assuming that the basis for analogue models is having an equation in common. As Table ZT.1 indicates, there are other bases for using analogue models than having an equation, and it is not the case that an analogue model can in principle always be replaced by an equation: the method of dimensional analysis and physically similar systems often requires less information than the method of finding an equation that is instantiated by both the analogue model and what it models does. (That is why similarity methods are sometimes called partial information methods. [Z.59] ) More importantly, even when one does have such an equation, and cites it as the basis for the analogy between analogue model and what is modeled, the role of the equation is to establish a correspondence between items in the analogue model and what it models. Even if one has the means to solve that equation numerically on a digital computer, there is no guarantee that the numerical solution of the equation will reveal every phenomenon that might be observed in the analogue model. It has been the case many times that the use of an analogue model shows a phenomenon that the numerical solution of the equation had not, no matter how many colorful visuals and graphics the computer program is capable of producing. Analogue models do need to be examined to determine when they are and are not appropriate for a certain investigation, but so, of course, do equations and numerical simulations. The fact that the use of analogue models in various fields has been revived (after supposedly being eliminated from those fields), often for new applications and employing new technologies, reflects something that is becoming increasingly clear: sometimes analogue models really are irreplaceable.

\section{Acknowledgements}

Thanks to Matt Walhout for suggesting, many years ago, that I might find William Unruh's work on sonic analogues of black holes of interest. Later, in April 2011, I had the good fortune to attend Unruh's lecture "Measurement of Hawking Radiation in an Analog System" and the discussion afterwards, at the University of Pittsburgh.

Thanks also to the organizers of the conference "Philosophy of Scientific Experimentation III" (PSX3), for financial support to present the talk this paper is based upon ("Experimentation on Analogues") at PSX3 on October 5, 2012, at the Department of Physics, University of Colorado, Boulder. I benefitted from comments by, and discussion with, the other participants of PSX3 on the many papers related to analogy, including James Mattingly's talk "Experimental Cosmology", which also discussed experimentation on an analogue model (using Bose-Einstein condensates), related to his published work in [Z.37].

Another paper on the topic of the same experiments on sonic analogues of black holes discussed in this paper [Z.54] [Z.55] was presented at the Philosophy of Science Association Biennual meeting in late 2014. As that presentation by Dardashti (and the subsequent publication 
of a related paper by Dardashti et al. [Z.60] ) occurred more than two years after I submitted and presented "Experimentation on Analogues" at PSX3 in October 2012, the talk on which my article for this volume is based, their commentary on those experiments is not discussed here.

\section{References}

Z.1 W. W. G. J. Swoboda: The thought and work of the young Ernst Mach and the antecedents to his philosophy (University of Pittsburgh, University of Pittsburgh Ph.D Dissertations, Pittsburgh PA 1973)

Z.2 John T. Blackmore: Ernst Mach: His Work, Life, and Influence (University of California Press, Berkeley and Los Angeles, CA 1972)

Z.3 Susan G Sterrett: Sounds Like Light: Einstein's Special Theory of Relativity and Mach's Work in Acoustics and Aerodynamics, Studies in the History and Philosophy of Modern Physics 29, 1 - 35 (1998)

Z.4 Pavel A. Cherenkov - "Nobel Lecture: Radiation of Particles Moving at a Velocity Exceeding That of Light, and Some of the Possibilities for Their Use in Experimental Physics". Nobelprize.org. Nobel Media AB 2014. Web. 2 May 2015. <http://www.nobelprize.org/nobel_prizes/physics/laureates/1958/cerenkov-lecture.html>

Z.5 C. Barcelo, S. Liberati, M. Visser: Analogue Gravity, Living Rev. Relativ., 14(3), 1 - 179 (2011)

Z.6 Matt Visser, Carlos Barcelo, and Stefano Liberati: Analogue Models of and for Gravity, General Relativity and Gravitation, 34 (10), 1719 -1734 (2002)

Z.7 Daniele Faccio: Laser pulse analogues for gravity and analogue Hawking radiation. Laser pulse analogues for gravity and analogue Hawking radiation, Contemporary Physics, 53:2, 97$112(2012)$

Z.8 Daniele Faccio, et.al (Eds.): Analogue Gravity Phenomenology: Analogue Spacetimes and Horizons from Theory to Experiment (Springer International Publishing, Switzerland 2013).

Z.9 Matt Visser: Survey of Analogue Spacetimes, Analogue Gravity Phenomenology: Analogue Spacetimes and Horizons from Theory to Experiment (Springer International Publishing, Switzerland 2013) pp. 31 - 50.

Z.10 lacopoc Carusotto and Germain Rousseaux: The Cerenkov Effect Revisited: From Swimming Ducks to Zero Modes in Gravitational Analogues, in Daniele Faccio, et.al (Eds.): Analogue Gravity Phenomenology: Analogue Spacetimes and Horizons from Theory to Experiment (Springer International Publishing, Switzerland 2013), pp. 109 - 144.

Z.11 Brown Ayres: Mechanical models of the electric circuit. The Electrical World (September 5, 1896), 28, 276-277 (1896)

Z.12 C. F. Jenkin: A dynamic model of tuned electrical circuits. in Proceedings of the Institution of Electrical Engineers, 60, 939 - 941. (1922) 
Z.13 James S. Small: The Analogue Alternative: The electronic analogue computer in Britain and the USA, 1930 - 1975, (Routledge, London and New York 2001)

Z.14 V. Bush and S. H. Caldwell: A New Type of Differential Analyzer, Journal of the Franklin Institute, 240 (4), 255 - 325. (1945)

Z.15 A. G. MacNee: An Electronic Differential Analyzer, Technical Report No. 90, Research Laboratory of Electronics, Massachusetts Institute of Technology. (1948)

Z.16 Brian Randell (Ed.): The Origins of Digital Computers: Selected Papers. (Springer Monographs in Computer Science, Springer-Verlag Berlin-Heidelberg 1982)

Z.17 Cyril Isenberg: The soap film: an analogue computer. American Scientist 64, 514-518. (1976)

Z.18 Masashi Aono, Yoshito Hirata, Maashiko Hara, Kazuyuki Aihara: Combinatorial Optimization by Ameoba-Based Neurocomputer with Chaotic Dynamics Natural Computing: Proc. 2nd International Workshop on Natural Computing, Nagoya, Japan, 2007. (Springer, printed in Japan 2009) pp. 1 - 15.

Z.19 S. G. Sterrett: Physically Similar Systems: A history of the concept, [this volume, chapter 2.]

Z.20 E. Buckingham: Physically Similar Systems: Illustrations of the use of dimensional equations, Physical Review, 4, 345-376. (1914)

Z.21 Henry L. Langhaar: Dimensional analysis and theory of models (Wiley, New York 1951)

Z.22 R. C. Pankhurst: Dimensional analysis and scale factors (Chapman and Hall, published on behalf of the Institute of Physics and the Physical Society, New York 1964)

Z.23 S. L. Lien and J. A Hoopes: Wind-driven, steady flows in Lake Superior, Limnology and Oceanography 23, 91 - 103. (1978)

Z.24 Nicholas P. Wallerstein, Carlos V. Alonso, Sean J. Bennett, and Colin R. Thorne: Distorted Froude-Scaled Flume Analysis of Large Woody Debris, Earth Surface Processes and Landforms 26, pp 1265 - $1283(2001)$

Z.25 S De Rosa, F Franco, and V Meruane: Similitudes for the structural response of flexural plates Proceedings of the Institution of Mechanical Engineers, Part C: Journal of Mechanical Engineering Science, first published February 17, 2015 (2015)

Z.26 Roman Frigg and Stephan Hartmann: Models in Science, The Stanford Encyclopedia of Philosophy (Fall 2012 Edition), Edward N. Zalta (ed.), URL = $<$ http://plato.stanford.edu/archives/fall2012/entries/models-science/>.

Z.27 Ronald N. Giere: Science without laws (University of Chicago Press, Chicago 1999)

Z.28 Ronald N. Giere: How models are used to represent reality, Philosophy of Science, 71, pp. 742-752. (2004) 
Z.29 Guala, Francesco (2002) "Models, Simulations, and Experiments" in Lorenzo Magnani and Nancy Nersessian (eds.), Model-Based Reasoning: Science, Technology, Values. New York: Kluwer, 59-74.

Z.30 Eric Winsberg: A tale of two methods, Synthese, 169 (3), 575-592. (2009)

Z.31 Daniel Rothbart (Ed.): Modeling: gateway to the unknown: a work by Rom Harre. (Studies in Multidisciplinarity) (Elsevier Science, Amsterdam, the Netherlands 2004)

Z.32 Peter Kroes: Structural analogies between physical systems, The British Journal for the Philosophy of Science, 40(2), 145-154. (1989)

Z.33 Edwin T. Layton: Escape from the Jail of Shape: Dimensionality and Engineering Science, in Peter Kroes and Martijn Bakker (Eds): Technological development and science in the Industrial Age: new perspectives on the science-technology relationship. (Kluwer Academic Publishers, Dordrecht; Boston 1992)

Z.34 S. G. Sterrett: Dimensional Analysis and Similarity, in A. W. M. Meijers (Ed.) Philosophy of technology and engineering sciences, Vol. 9, p.799 - 823 (North Holland/Elsevier, Amsterdam, The Netherlands 2009)

Z.35 Susan G. Sterrett: Wittgenstein Flies A Kite: a story of models of wings and models of the world. (Pi Press/Penguin, New York 2005/6)

Z.36 Susan G. Sterrett: Physical models and fundamental laws: using one piece of the world to tell about another, Mind and Society, 5 (3), 51-66. (2002)

Z.37 James Mattingly and Walter Warwick: Projectible predicates in analogue and simulated systems, Synthese, 169, 465-483 (2009)

Z.38 Sjoerd D. Zwart: Scale modelling in engineering: Froude's case, in A. W. M. Meijers (Ed.) Philosophy of technology and engineering sciences, Vol. 9, p.759 - 798 (North Holland/Elsevier, Amsterdam, The Netherlands 2009)

Z.39 Naomi Oreskes: From Scaling to Simulation: Changing meanings and ambitions of models in geology. In Angela N. H. Creager, Elizabeth Lunbeck, and M. Norton Wise (Eds.): Science Without Laws (Duke University Press, Durham, London 2007)

Z.40 M, King Hubbert: Theory of scale models as applied to the study of geologic structures, The Geological Society of American Bulletin, 48, pp. 1459 - 1520. (1937)

Z.41 M. King Hubbert: Strength of the Earth. Bulletin of the American Association of Petroleum Geologists, 29, 1630 - 1653 (1945)

Z.42 Olivie Merle and Andrea Borgia: Scaled experiments of volcanic spreading, J. of Geophys. Res. Solid Earth, 101, 13805-13817 (1996)

Z.43 Analogue modeling of flank instability at Mount Etna: Understanding the driving factors, J. Geophys. Res., 116, 1-21.

Z.44 Vahe Gabuchian, Ares J. Rosakis, Nadia Lapusta, and David D. Oglesby: Experimental investigation of strong ground motion due to thrust fault earthquakes, J. of Geophys. Res. Solid Earth, 119, 1316-1336 (2014) 
Z.45 Hermann von Helmholtz: On Discontinuous Motions in Liquids, Mechanics of the Earth's Atmosphere: A Collection of Translations by Cleveland Abbe. Smithsonian Miscellaneous Collections No. 843, 58 - 66 (The Smithsonian Institution, Washington DC 1891)

Z.46 Hermann von Helmholtz: On a Theorem Relative to Movements that are Geometrically Similar in Fluid Bodies, Together with an Application to the Problem of Steering Balloons, Mechanics of the Earth's Atmosphere: A Collection of Translations by Cleveland Abbe.

Smithsonian Miscellaneous Collections No. 843, 67 - 77 (The Smithsonian Institution, Washington DC 1891)

Z.47 W. G. Unruh: Experimental Black-Hole Evaporation?, Phys. Rev. Lett., 46, 1351- 1353 (1981)

Z.48 Richard Courant and K. O. Friedrichs: Supersonic flow and shock waves (Springer, New York 2012 (reprint of original 1948 edition))

Z.49 Alberto Bramati and Michele Modugno (Eds.): Physics of quantum fluids: new trends and hot topics in atomic and polariton condensates (Springer, Heidelberg 2013)

Z.50 Max Born: Einstein's Theory of Relativity (Dover Books, New York 1962)

Z.51 Ralf Schutzhold and William G. Unruh: Gravity wave analogues of black holes, Phys. Rev. D, 66, 044019 (2002)

Z.52 W. G. Unruh: Dumb Holes: analogues for black holes, Phil. Trans. R. Soc. A, 366, 2905$2913(2008)$

Z.53 Matt Visser: Hawking radiation without black hole entropy, Phys. Rev. Lett., 80 (16), 3436(1998)

Z.54 Silke Weinfurtner, Edmund W. Tedford, Matthew C. J. Penrice, William G. Unruh, and Gregory A. Lawrence: Measurement of stimulated Hawking emission in an analogue system, Physical Review Letters, 106, 021302-021305 (2011)

Z.55 Silke Weinfurtner, Edmund W. Tedford, Matthew C. J. Penrice, William G. Unruh, and Gregory A. Lawrence: Classical aspects of Hawking radiation verified in analogue gravity experiment, in Daniele Faccio, et.al (Eds.): Analogue Gravity Phenomenology: Analogue Spacetimes and Horizons from Theory to Experiment (Springer International Publishing, Switzerland 2013), pp. 167-180.

Z.56 Germain Rousseaux: The Basics of Water Waves Theory for Analogue Gravity, in Daniele Faccio, et.al (Eds.): Analogue Gravity Phenomenology: Analogue Spacetimes and Horizons from Theory to Experiment (Springer International Publishing, Switzerland 2013) pp. 81-107.

Z.57 Matt Visser: Essential and inessential features of Hawking radiation, Int. J. Mod. Phys. D, $12,649-661$ (2003)

Z.59 Stephen J. Kline: Similitude and approximation methods (Springer Verlag, 2012)

Z.60 Radi Dardashti, Karim Thebault, and Eric Winsberg: Confirmation via analogue simulation: what dumb holes could tell us about gravity (forthcoming in British Journal for the Philosophy of Science 2015) 